\title{
A Study on Underwater based Wireless Sensor Networks
}

\author{
Aiman J. Albarakati \\ Department of Computer Engineering, \\ College of Computer and Information Science, Majmaah University, \\ Majmaah, Saudi Arabia
}

\begin{abstract}
A lot of researchers lately have attained the attention of Wireless Sensor Networks. On the other hand, when measures it up to the terrestrial or global WSN, Underwater Sensor Networks (UWSN) depicts an innovative networking standard. The exploitation of Underwater Sensor Networks is not simple or clear-cut, fundamental defies are therefore required to be mapped just because the sort of settings of underwater. This research article has illustrated a contrast between two sorts of sensor networks. Furthermore we also discussed the vital defies, facets of Underwater sensors comprising their protocols and architectures and future research prospects.
\end{abstract}

Keywords: WSN, Underwater Sensors, Communication Protocols, Sensor Networks

\section{INTRODUCTION}

Approximately $70 \%$ of our earth/world is covered by water. As a lot of research is being progressed on underwater systems, data collection and atmosphere monitoring turn out to be actually most important competitors. This heaves the call for a successful approach to gather data and keep an eye on the surroundings or concern settings. UWSN puts forward a consummate alternative. The distinctiveness of the underwater surroundings depict researchers with a lot of defies above all, successful communication and sensor localization methods. In terrestrial WSN, the nodes bring into play radio frequency (RF) to put up together the communication. In underwater surroundings, due to water assimilation, radio doesn't function fit. Measure up to to radio waves; sound has advanced proliferation distinctiveness in water, crafting it the favored technology for underwater communications. By and large, radio waves disseminate at extensive detachments all the way through accomplished seawater merely at superfluous squat frequencies around at $30-300 \mathrm{~Hz}$, which entail hefty or big antenna and elevated transmission power. Optical waves don't undergo with such towering dwindling but are exaggerated by dispersion. Furthermore, communication of optical signals entails towering exactitude in spotting slender laser beams [1]. In consequence, nearly all underwater networks utilize acoustic/auditory signals to be in touch to each other.

\section{ACOUSTIC BASED UNDERWATER WSN's}

Various factors affect underwater acoustic dissemination that formulates scheming an underwater WSN exigent. Following are the factors:

- Bandwidth: There is restricted acoustic band under the water just because of assimilation; nearly all acoustic systems maneuver fewer than or under $30 \mathrm{kHz}$. [2]
Illustrated that no such research or viable system can surpass $40 \mathrm{~km} \times \mathrm{kb} / \mathrm{s}$ as the upper limit manageable range $\times$ rate product.

- Dissemination delay: The alacrity of Radio Frequency is $3 \times 10^{8} \mathrm{~m} / \mathrm{s}$ whereas the acoustic signal dissemination tempo is $1.5 \times 10^{3} \mathrm{~m} / \mathrm{s}$ in an underwater. The propagation/dissemination delay in underwater is five orders of extent superior than in Radio Frequency. The squat pace of sound gives an invitation to multi-path dissemination to draw out over time delay. It significantly upshots the synchronized application of Underwater Sensor Networks.

- Shadow zones: It is an area where we will have soaring bit error rates and provisional fatalities of connectivity just because of intense distinctiveness of the underwater channel. Density, Salinity and temperature disparities of the water can manipulate acoustic transmission, like provisional fatalities of connectivity [3].

- Energy: The power of the Batteries is restricted for the reason that these batteries are awfully thorny to recharge or revive.

- $\quad$ Failure: These sensors are flat to malfunction due to stinking and decomposition.

- Attenuation: attenuation is the diminution in amplitude and intensity of a signal [4].

\section{DIVERSITIES}

Even though Underwater Sensor Networks and Wireless Sensor Networks are dissimilar just because of the distinctive individuality of water, there is a great chance pf applying convinced facets of Wireless Sensor Networks research to Underwater Sensor Networks. Following are the core diversities or dissimilarities between underwater sensor networks and terrestrial:

1. Communication technique: Underwater Sensor Networks utilizes acoustic signal whereas Wireless Sensor Network employs radio waves.

2. Outlay: Despite the fact that terrestrial sensor nodes are predictable to befall ever more economical whereas underwater sensors are costly devices. It is just because of the Underwater Sensor Networks transceivers intricacy and the improved fortification hardware obligation.

3. Power: Underwater Sensor Networks entails more power for the reason that it utilizes acoustic signal and cover up a longer distance. In contrast with the acoustic signal, Radio 
Frequency desires less power, in view of the fact that the dispensation at receivers is not that multifaceted.

4. Memory: The link of an acoustic signal is able to put out of action by an unusual underwater state of affairs, like shadow zones. In view of that fact, underwater sensors have to get hold of more data to thwart the failure of data. Nevertheless, terrestrial sensors don't have problem.

5. Density: Likewise sensors are able to be employed compactly in a terrestrial sensor application. At the same time as an underwater sensor is more luxurious than terrestrial sensor, it will outlay more capital to set up tightly. yet if money is not a concern, it is still not trouble-free to position them.

Actually, those discrepancies are the inklings to build up novel generation Underwater Sensor Networks. Foremost, we ought to engender a number of up-to-the-minute kinds of sensors to trim down outlay. E.g. we can make use of nanotechnology to build up nano-sensors. Furthermore, it is obligatory to work out periodical clean-up methods aligned with corrosion and fouling, which may possibly bang the life span of underwater devices. In addition, the employed network has to be exceedingly steadfast, so as to steer clear of malfunction of monitoring operations just because breakdown of solitary or manifold sensors. Subsequent, we require doing an innovative power control algorithm for Underwater Sensor Networks. Several multifaceted power control algorithm utilizing RTS-CTS-ACK have been projected in ancient times for wireless terrestrial networks [5][6]. Nevertheless, these algorithms cannot on top form into Underwater Sensor Networks just because underwater channel distinctiveness and considerable dissemination delays. Third, network protocol is a significantly main aspect in saving power and endowing with dependable connection utilizing meager underwater sensors. At the moment, loads of unusual protocols for terrestrial Wireless Sensor Networks have been systemized. Nonetheless, they cannot on top form for Underwater Sensor Networks. The architectures of Underwater Sensor Networks are not the only aspect that blows the progress of a novel protocol, but distinctiveness of underwater too. It is a new diverse set with terrestrial sensor network. As a result, we may possibly build up dissimilar sorts of protocol with respect to the architectures of Underwater Sensor Networks.

\section{STRUCTUAL DESIGN OF UWSN}

[7] Illustrated that Underwater Sensor Networks can be more or less categorized into two wide-ranging categories: longterm non-time-critical aquatic monitoring/observation and short-time time-critical aquatic exploration.

According to [1], Underwater Sensor Networks are categorized into three types: Static two-dimensional underwater acoustic sensor networks (UW-ASNs) which are used to monitor the bottom of an ocean.

Static three-dimensional UW-ASNs which are bring into play to keep an eye on ocean-column. Three dimensional networks of autonomous underwater vehicles (AUVs).

\subsection{Short-Term Time-Critical Aquatic Exploration/monitoring}

In contrast to other methods this method spotlight on instantaneous data. For that reason, how to construct data transmit competently have to be more distress when scheming network protocol. Also, this sort just exert for a small term that means energy saving is not as imperative as others.

\subsection{Long-Term Non-Time-Critical Aquatic Monitoring/exploration:}

This approach is efficient for an elongated time and the data collected/gathered by the sensors are not synchronized data. For long-term monitoring, energy saving is an innermost concern to mull over in the protocol devise.

\subsection{Assessment of the Two Classifications:}

The divergence between the two classifications is static and mobile. According to [7], long-term non-time-critical and short-term time-critical Underwater Sensor Networks are mobile aptitude supported. For this reason they distress the position aware in whichever way. Furthermore, long-term and short-term didn't discriminate 2D or 3D. Evidently, there are a number of discrepancies in protocol devise. All the sensors are anchored to the underside of ocean in a motionless/static two-dimensional. These sensor nodes are unified to one or more underwater sinks (uw-sinks) by acoustic signal. In view of the fact that, all the sensors are rigid at bottom, for that reason, we don't require to unease underwater faction, which formulate protocol devise effortlessly. 2D UW-ASNs for all time are utilized to ecological monitoring; it has to to be sort of long-term nontime-critical Underwater Sensor Network. Consequently, defies of long-term non-time critical Underwater Sensor Network also in shape it. Static three-dimensional underwater sensor network, measure up to two-dimensional one, this category as well tells the profundity in order to cover up 3D region. It is hard to design the protocol for three-dimensional UWSN. The pace and dissemination delay of acoustic signal is unusual at unlike strength which will construct a number of sensors at convinced strength utilize more energy to propel and take delivery of data. In addition, this subject constructs it stiff to construct up a competent routing. One more defy in this structural design is how to craft the sensor hang about at stick spot. The most recent one, three-dimensional networks of autonomous underwater vehicles (AUVs), is able be looked upon as long-term or short-term. And one fundamental central devise intention is to formulate them rely on local astuteness and not as much of reliant on communications from online shores. According to [1] even though, we require doing the protocol with respect to the unusual application, we still call for spotlight on a number of widespread necessities, e.g. protection, dependable and elasticity.

\section{RESEARCH PROSPECTS \\ 5.1 Power Consumption or Utilization}

Contrasting the sensors of terrestrial Wireless Sensor Network, Underwater Sensor Networks sensor is not capable to utilize solar energy to revitalize the battery. And it is extremely tricky to put back the sensors. The express line of attack to make out a way for such dilemma is to engender energy by the sensors by their own. The feasible scheme may possibly be employing in progress faction or chemistry process to produce power to recharge or revitalize battery. In addition, well-organized routing protocol and communication process is able to put in to this matter.

\subsection{Communication Link}

These days, most of Underwater Sensor Networks sensor bring into play acoustic signals to be in touch. Acoustic signals carry lots of defies to the research field, above all propagation or proliferation delay and elevated error rates. Perceptibly, it calls for enhancement. For that reason, endeavoring to bring into play a different sort of signal may possibly be a novel course of research. According to [8], 
optical signals have been employed to commune sensors in their two applications. It may possibly be an unusual method. Nonetheless, it requires mulling over all the aspects, more than ever power problem, to settle on whether optical signal is superior than acoustic one.

\subsection{Distributed Localization and Time harmonization or Synchronization}

Location-aware is significantly an imperative aspect for any aquatic/water application. In view of the fact that the data with no position information is a waste of time. Amongst a large amount of extensive terrestrial Wireless Sensor Network application, GPS can be utilized to bestow the position and coordinate time. Time-Differenc-of-Arrival (TDoA) In the GPS-free terrestrial application is utilized to compute the distance with respect to the unusual speed/pace of the two signals. Then the location/position information will be able to premeditated/computed employing those distance data by a number of algorithms similar to SemiDefinite Programming (SDP) [9]. In Underwater Sensor Network, the location information is bale to be computed utilizing identical way. Yet, the defy is that it is very rigid to acquire the distance amid two sensors. GPS is not able to be utilized, in view of the fact that the satellite signal cannot exert in underwater. Even though, a number of schemes are brought into play to dig up the distance between the sensors, like Time-of-Arrival (ToA), Angle-of-Arrival (AoA) the truthfulness is seriously exaggerated by loads of aspects of underwater setting. For that reason, how to get a hold of an exact distance between two sensors should acquire more distress in order to search out underwater sensors' location.

\subsection{Routing Protocols}

Saving energy is an extremely important factor in the scheming Underwater Sensor Network protocol design, in particular for the long-term aquatic monitoring applications. In point of fact, that there are abundant terrestrial Wireless Sensor Network energy-proficient protocols being fashioned in this region/field. On the other hand, due to the node mobility of underwater sensor network, nearly all of them are not realistic in underwater sensor network, In view of the fact that structural design of underwater sensor network bestows more blows measure up to the terrestrial one. As a result, structural design ought to bestow more concentration when routing protocol is going to be designed.

\section{CONCLUSION}

Underwater wireless sensor networks (UWNS) will turn out to be more and more imperative on the study of underwater planet. We discussed the exclusive distinctiveness of the underwater surroundings and its outcome on the devise of underwater sensor network. Moreover, the divergence between terrestrial Wireless Sensor Network and underwater sensor network are illustrated. Although they are unusual, terrestrial Wireless Sensor Network is at a standstill priceless on underwater sensor network. Most important defies comprising power burning up, communication practices, and routing protocols are demonstrated. These facets are very important important to do prospect research on underwater sensor network, in particular crafting novel generation underwater sensor network. It is obvious that the call for for novel/innovative location-aware communication protocols is not able to be miscalculated. Even in various cases, locationaware capability is the most significant aspect match up to others in underwater sensor network application.

\section{REFERENCES}

[1] Akildiz, I.F., Pompili, D., Underwater Acoustic Sensor Networks: Research Challenges, Ad Hoc Networks,3, (260), 2005.

[2] Kifoyle, D.B., The State of the Art in Underwater Acoustic Telemetry, IEEE J. Oceanic Eng., , Jan. 2000.

[3] Etter, P. C., Underwater Acoustic Modeling, Principles, Techniques and Applications. 2nd edition, E\&FN Spon, 1996.

[4] Berkhovskikh, L., Lysanov, Y., Fundamentals of Ocean Acoustics, New York: Springer, 1982.

[5] Proakis, J., Rice, J.,., Shallow water acoustic networks, Encyclopedia of Telecommunications, Proakis, J. G., Ed. John Wiley and Sons, 2003.

[6] Knudsen, V.O.,., Digital Communications. Marine Research (7-12), 410, 1948.

[7] Cui, J.H., Kong, J.,., The Challenges of Building Scalable Mobile Underwater Wireless Sensor Networks for Aquatic Applications, IEEE Network, (0890-8044), 12-17, May/June 2006.

[8] Vasilescu, I., Kotay, K.,: An Exploration in Underwater Sensor Networks, Second IEEE Workshop, (30-31), 151152, May 2005.

[9] Biswas, P., Semidefinite programming for ad hoc wireless sensor network localization, Proceedings of the third international symposium on Information processing in sensor networks, (46-54), April 2004. 